\title{
A NASH-MOSER FRAMEWORK FOR FINDING PERIODIC SOLUTIONS OF THE COMPRESSIBLE EULER EQUATIONS
}

\author{
BLAKE TEMPLE AND ROBIN YOUNG
}

\begin{abstract}
In this paper, which summarized a talk given by the second author in Waterloo, reports on recent progress the authors have made in a long term program to prove the existence of time-periodic shockfree solutions of the compressible Euler equations. We briefly recall our previous results, describe our recent change of direction, and discuss the estimates that must be obtained to get the Nash-Moser method to converge. Assuming these estimates, we present a new convergence theorem for the Nash-Moser method. Our approach reduces the problem to that of obtaining small divisor estimates for finite dimensional projections of linearized operators. These operators can be described in detail, and in principle, this reduces the proof of periodic solutions to the calculation of the smallest singular value of an $N \times N$ matrix.
\end{abstract}

\section{INTRODUCTION}

We outline our program for proving the existence of nontrivial time- and space-periodic solutions to the compressible Euler equations,

$$
\begin{aligned}
\tau_{t}-u_{x} & =0 \\
u_{t}+p_{x} & =0 \\
\left(\frac{1}{2} u^{2}+e\right)_{t}+(u p)_{x} & =0,
\end{aligned}
$$

with $p=K e^{S / c_{\tau}} \tau^{-\gamma}, e=p \tau /(\gamma-1)$.

These equations model an inviscid, $\gamma$-law gas in Lagrangian frame, in one space dimension. Since this a nonlinear hyperbolic system, global classical solutions generally exist only for short times, after which shocks form and weak entropy solutions need to be considered. There are now mature theories for the existence, uniqueness and $L^{1}$-stability of such systems in the class of solutions having small total variation $[4,16,1,2]$.

The presence of shocks in solutions is stabilizing, in that solutions with shocks necessarily decay due to the entropy conditions. Riemann first showed that shocks necessarily form in the isentropic $(2 \times 2)$ equations [10], and the corresponding decay theory was settled in the celebrated work of Glimm and

Temple supported in part by NSF Applied Mathematics Grant Number DMS-040-6096. Young supported in part by NSF Applied Mathematics Grant Number DMS-010-4485. 
Lax [5]. In the isentropic system, shocks necessarily form for all nontrivial data of any size, and the variation of spatially periodic solutions decays like $1 / t$. For $3 \times 3$ systems, it has been shown that shocks form for any compactly supported initial data having small total variation $[6,7]$. Less is known when the data has large total variation, and in particular for spatially periodic data, for which large-time but not global existence is known [12].

In a series of papers, the authors discovered a mechanism for the delay or prevention of shock formation in the full $3 \times 3$ Euler equations (1) $[13,15,14]$. This is an effect of multiple wave interactions, in which a compression wave can change its character and become a rarefaction as it crosses a contact discontinuity, provided the opposite wave has the appropriate sign and strength. If this type of interaction occurs in a periodic manner, a periodic pattern of waves could be set up in which compressions and rarefactions continually balance, yielding a nontrivial wave structure which is periodic in both space and time, and has no shocks. This wave structure is described in [13], and the corresponding linearized problem is studied in [15].

In our ongoing work, the authors are attempting to make this construction rigorous by proving a perturbation theorem via the Nash-Moser method. In this paper, we outline our current strategy for completing this program by describing the Nash-Moser iteration and highlighting the estimates that will need to be proved to complete the proof of existence of periodic solutions. We trust that this also provides a useful exposition of the power and use of the Nash-Moser method.

We begin by briefly describing the fully nonlinear problem after nondimensionalization and vectorization, and rewriting it in an abstract form suitable for study by the Nash-Moser method. In Section 3, we describe the abstract Nash-Moser Newton iteration that we will use, and in Section 4 we describe the specific norms and smoothing operators that seem to be best suited to the problem. In Section 5, we explicitly state the bounds and estimates that are necessary to complete our proof of existence, and in Section 6 we prove convergence of the iteration, assuming those estimates. This implies that establishing the estimates in Section 5 would complete the first proof of existence of periodic solutions of compressible Euler.

As a final comment, in a nutshell, our recent new approach is to address the issue of resonances in a conservative framework sufficient to obtain small divisor estimates for finite dimensional approximations of the linearized operators that appear in the Newton method. This follows more closely the philosophy of Nash-Moser set out in the early work of Moser [9], (with the added feature of expunging resonant periods), and responds to our earlier failed attempts at obtaining small divisor estimates in an infinite dimensional setting, c.f. [3]. 


\section{Nonlinear PROBlem}

The solutions described in [13], which exhibit a periodic wave structure, consist of balanced rarefaction and compressive sound waves interacting with a stationary entropy profile which consists of a periodic square wave. This square wave can be characterized by the widths of the different entropy levels and the size of the entropy jump (or ratio of values). Our nonlinear problem is to then resolve the forward and backward waves which are to be superimposed on this entropy profile.

In [15], the authors make several simplifications. The first is to change the sense of the independent variables, so that the solution evolves in space rather than time. We then perform a non-dimensionalization and a vectorization, so that the fully nonlinear problem can be succinctly expressed.

Once the entropy profile is fixed as a square wave, and because we are considering solutions without shocks, away from the entropy jumps, the equations reduce to those of isentropic gas dynamics, the so-called $p$-system. After non-dimensionalizing, and written in quasilinear form, this becomes the system

$$
\begin{aligned}
p_{x}+v_{t} & =0 \\
v_{x}-q(p)_{t} & =0,
\end{aligned}
$$

where $v$ is the rescaled fluid velocity, $q(p)=\gamma p^{-1 / \gamma}$ is the rescaled specific volume, and we regard the (rescaled) spatial coordinate $x$ as the evolution variable. We denote the nonlinear evolution operator through $\theta$ by $\mathcal{E}(\theta)$, so that

$$
\mathcal{E}(\theta)(U(0, \cdot))=U(\theta, \cdot),
$$

where $U(x, t)=(p, v)(x, t)$ solves $(2)$.

Equations (2) hold for both values taken on by the entropy, and the effect of crossing a contact discontinuity of strength $J$ is to scale $v$ by $J$, while leaving $p$ unchanged: that is,

$$
\left[\begin{array}{l}
p_{+} \\
v_{+}
\end{array}\right]=\mathcal{J}\left[\begin{array}{l}
p_{-} \\
v_{-}
\end{array}\right], \quad \text { where } \mathcal{J} \equiv\left(\begin{array}{ll}
1 & 0 \\
0 & J
\end{array}\right),
$$

the subscripts denoting the states on opposite sides of the entropy jump; note that $\mathcal{J}$ is a diagonal linear operator.

In these non-dimensional variables, the square entropy wave is fully described by parameters $J, \bar{\theta}$ and $\underline{\theta}$, which are, respectively the size of the jump and the widths of the different entropy levels. Then according to $[13,15]$, our nonlinear problem is to find $2 \pi$-periodic functions $U(\cdot)=(p, v)$ and parameters for which

$$
\mathcal{S} \mathcal{J}^{-1} \mathcal{E}(\underline{\theta}) \mathcal{J} \mathcal{E}(\bar{\theta})(U)=U,
$$

where $\mathcal{S}$ is a half-period shift, $\mathcal{S}(u(t))=u(t-\pi)$.

It is convenient to take $\bar{\theta}=\underline{\theta}$ and vectorize the problem: doing so, $U$ becomes a $4 \times 4$ vector, $U=(\bar{p}, \bar{v}, \underline{p}, \underline{v})^{T}$, the components corresponding to 
the two different entropy levels. We define the $4 \times 4$ operators

$$
\hat{\mathcal{E}}(\theta)=\left(\begin{array}{ll}
\mathcal{E} & 0 \\
0 & \mathcal{E}
\end{array}\right) \quad \text { and } \quad \hat{\mathcal{J}}=\left(\begin{array}{cc}
0 & \mathcal{J}^{-1} \\
\mathcal{S} \mathcal{J} & 0
\end{array}\right)
$$

where $\mathcal{E}=\mathcal{E}(\theta)$ is given by (3) and $\mathcal{J}$ by (4).

With this notation, our fully nonlinear problem can be written

$$
\mathcal{F}(U) \equiv \hat{\mathcal{E}}(\theta)(U)-\hat{\mathcal{J}} U=0,
$$

which can be regarded as a $4 \times 4$ problem with $2 \times 2$ blocks. The advantage of this formulation is that the parameters $\theta$ and $J$ are well separated, and indeed the only nonlinear part of $\mathcal{F}$ is in the $2 \times 2$ evolution blocks $\mathcal{E}$, as $\hat{\mathcal{J}}$ is linear.

The constant state $\mathbb{1} \equiv(1,0,1,0)^{T}$ is an exact solution to $(5)$, and we wish to perturb this to get a nontrivial solution of the nonlinear problem. Linearizing (2) around $\mathbb{1}$ yields the linear wave equation. This in turn respects Fourier modes, as does $\mathcal{J}$, which allows us to give a complete description of the kernel of the linearized operator as a direct sum of $4 \times 4$ matrix problems, see [15]. In particular, this linearized operator admits a 1-mode solution $Z=Z(x, t)$ if and only if

$$
J=\frac{1+\cos \theta}{1-\cos \theta} \quad \text { or } \quad J=\frac{1-\cos \theta}{1+\cos \theta} .
$$

More generally, the linearized operator has an $n$-mode solution if and only if

$$
J=\frac{1+\cos (n \theta)}{1-\cos (n \theta)} \quad \text { or } \quad J=\frac{1-\cos (n \theta)}{1+\cos (n \theta)} ;
$$

it follows that if $\theta$ is a rational multiple of $\pi$, then there are resonances, while if not, there are small divisors. Thus we cannot apply the Implicit Function Theorem, and we resort to the Nash-Moser method.

\section{NASH-MOSER ITERATION}

We recall the Nash-Moser iteration method [9]. This is a modified Newton's method for solving the equation $\mathcal{F}(U)=0$, applied to problems in which Newton's method does not apply, such as those with small divisors, loss of derivatives, or both.

First, we recall Newton's method. To this end, suppose $U_{0}, \ldots, U_{m}$ have been defined: set

$$
U_{m+1}=U_{m}-X_{m+1},
$$

where $X_{m+1}$ is defined by

$$
D \mathcal{F}\left(U_{m}\right)\left[X_{m+1}\right]=Y_{m}, \quad \text { with } \quad Y_{m}=\mathcal{F}\left(U_{m}\right) .
$$

Then we estimate

$$
Y_{m+1}=\mathcal{F}\left(U_{m+1}\right)-\mathcal{F}\left(U_{m}\right)+D \mathcal{F}\left(U_{m}\right)\left[X_{m+1}\right]=O\left(\left\|X_{m+1}\right\|^{2}\right),
$$


where we have used the Taylor expansion for $\mathcal{F}$. Thus if the inverse of the linearized map $D \mathcal{F}$ is bounded, we get

$$
\left\|Y_{m+1}\right\| \leq K\left\|Y_{m}\right\|^{2}
$$

that is, quadratic convergence to the root, provided our initial guess $U_{0}$ is close enough.

The idea of Nash-Moser is to modify the iteration by mollifying at each step, in such a way that the approximations remain bounded. Then the iteration can be defined at each step, and the smoothing is weakened so that less and less smoothing is introduced at each step, but the higher order convergence from Newton compensates for the ensuing loss of smoothness, so that convergence can be proved as long as $U_{0}$ is close enough to the root and certain compatibilty conditions are met.

We now write down the abstract Nash-Moser iteration in detail. At the $m$-th step, we approximate our fully nonlinear function $\mathcal{F}$ by the approximation $\mathcal{F}_{m}$; we can also approximate the derivative $D \mathcal{F}_{m}$ by a modified linear operator $\mathcal{A}_{m}$, if necessary.

If the iterates $U_{0}, \ldots, U_{m}$, together with associated residuals $Y_{j}$ and differences $X_{j}$ have been defined, we set

$$
Y_{m}=\mathcal{F}_{m}\left(U_{m}\right)
$$

where $\mathcal{F}_{m}$ is the $m$-th (mollified) approximation to $\mathcal{F}$, and solve the linear equation

$$
\mathcal{A}_{m}\left[X_{m+1}\right]=Y_{m}
$$

where $A_{m}[\cdot] \approx D \mathcal{F}_{m}\left(U_{m}\right)[\cdot]$ is an approximation of the derivative $D \mathcal{F}_{m}$, evaluated at $U_{m}$. Finally we update the iteration by setting

$$
U_{m+1}=U_{m}-X_{m+1} .
$$

Using (8), (9) and (10), we now write

$$
\begin{aligned}
Y_{m+1}= & \mathcal{F}_{m+1}\left(U_{m+1}\right)-\mathcal{F}_{m}\left(U_{m+1}\right) \\
& \quad+\mathcal{F}_{m}\left(U_{m}-X_{m+1}\right)-\mathcal{F}_{m}\left(U_{m}\right)+\mathcal{A}_{m}\left[X_{m+1}\right] \\
=I & +I I+I I I
\end{aligned}
$$

where we have set

$$
\begin{aligned}
I & =\mathcal{F}_{m+1}\left(U_{m+1}\right)-\mathcal{F}_{m}\left(U_{m+1}\right), \\
I I & =\mathcal{A}_{m}\left[X_{m+1}\right]-D \mathcal{F}_{m}\left(U_{m}\right)\left[X_{m+1}\right], \quad \text { and } \\
I I I & =\mathcal{F}_{m}\left(U_{m}-X_{m+1}\right)-\mathcal{F}_{m}\left(U_{m}\right)+D \mathcal{F}_{m}\left(U_{m}\right)\left[X_{m+1}\right] .
\end{aligned}
$$

We interpret each of these as follows: $I$ is the difference in smoothing between the $m$-th and $(m+1)$-st steps; $I I$ is the error in approximating the linearized operator; and III is the (quadratic) error from the Taylor expansion of $\mathcal{F}_{m}$.

If we can find appropriately small bounds for $I$ and $I I$, and if in addition we can find a bound for $X_{m+1}$ in terms of $Y_{m}$ in (9), then the quadratic Taylor estimate of III means that the iteration will again converge, despite 
the presence of small divisors and/or loss of derivatives. This is the sense in which the Nash-Moser iteration allows for derivative loss in each of the above steps.

To carry out this program and prove existence of a solution of the (infinite dimensional) equation $\mathcal{F}(U)=0$, we need the following ingredients:

- An appropriate choice of norms;

- A choice of mollified operators $\mathcal{F}_{m}$;

- A quadratic Taylor estimate for each $\mathcal{F}_{m}$;

- Convergence with a rate of the operators $\mathcal{F}_{m} \rightarrow \mathcal{F}$;

- A good approximation $\mathcal{A}_{m}$ of the linearization $D \mathcal{F}_{m}\left(U_{m}\right)[\cdot]$;

- A bound for the inverse $\mathcal{A}_{m}^{-1}$; and

- A good initial guess.

In general, the operator $\mathcal{F}$ may also depend on one or more parameters, denoted $\Theta$. Often, $\Theta$ will be a bifurcation parameter which encodes resonances in the fully nonlinear problem. In our case, $\Theta=(\theta, J)$ represents the nondimensionalized width of entropy levels and size of the entropy jumps.

When there is a parameter dependence in the problem, such as in our case, it may be that all of the forward estimates (i.e. estimates on $\mathcal{F}_{m}, \mathcal{A}_{m}$ ) are uniform in the parameter, but a uniform inverse estimate for $\mathcal{A}_{m}^{-1}$ is not available due to resonances. In this case, we can introduce another step into the Nash-Moser iteration, namely the expunging of resonant parameter values. That is, at the $m$-th step, we exclude a set $\mathcal{N}_{m}$ of $\Theta$ s of small measure, off of which we do obtain bounds for $\mathcal{A}_{m}^{-1}$. Restricting the set of parameter values in this way at each step, we obtain convergence of the iteration for $\Theta$ outside the set $\cup \mathcal{N}_{m}$, which does not have full measure.

\section{Norms And Smoothing}

Our nonlinear operator $\mathcal{F}=\hat{\mathcal{E}}-\hat{\mathcal{J}}$ consists of evolution in the $p$-system (2) and a bounded linear matrix operator (4), which is bounded on any space using any reasonable norm. We therefore choose the space and norm appropriate for a nonlinear hyperbolic system. It is well known that these systems are locally well-posed in the Sobolev spaces $H^{s}$, for $s>1 / 2$, [8]. We

need not consider large-time behavior because we are interested in solutions before the formation of shocks, i.e. gradient blowup.

Recalling that $x$ is the evolution variable in (2), it is convenient to express $(p, v)$ and $q(p)$ as Fourier series, so we write

$$
\begin{aligned}
& p(x, t)=\sum p_{k}(x) e^{i k t}, \\
& v(x, t)=i \sum v_{k}(x) e^{i k t}, \quad \text { and } \\
& q(x, t)=\sum q_{k}(x) e^{i k t},
\end{aligned}
$$

where the extra $i$ on $v$ is a convenience to ensure the equations for the coefficients are real. Plugging these into equations (2) and assuming convergence 
as necessary, our PDE (2) can be considered as an infinite system of ODEs, namely

$$
p_{k}^{\prime}=k v_{k}, \quad v_{k}^{\prime}=k q_{k},
$$

which is closed since we regard

$$
q_{k}(x)=q_{k}(P(x))=q_{k}\left(\ldots, p_{j}, \ldots\right),
$$

these being the Fourier coefficients of $q(p)=\gamma p^{-1 / \gamma}$. We can formally write this system in matrix notation, as follows. We set

$$
P(x)=\left\langle p_{j}(x)\right\rangle, \quad V(x)=\left\langle v_{j}(x)\right\rangle, \quad \text { and } \quad Q(x)=\left\langle q_{j}(x)\right\rangle,
$$

the indices ranging over all of $\mathbb{Z}$, and define the constant differentiation matrix

$$
\boldsymbol{D}=\operatorname{diag}\{\mathbb{Z}\}=\left[\delta_{j k}\right]= \begin{cases}1, & j=k, \\ 0, & j \neq k\end{cases}
$$

Then we can write the system as

$$
P^{\prime}=\boldsymbol{D} V, \quad V^{\prime}=\boldsymbol{D} Q(P),
$$

where these are infinite matrices and convergence of sums is assumed.

We can thus regard (2) as the system of ODEs (14) in the coefficient vectors $(P, V)$, which are sequences indexed by $k \in \mathbb{Z}$. The evolution operator $\mathcal{E}(\theta)$ is then integration of (14) from $x=0$ to $x=\theta$. The jump operator $\mathcal{J}$ respects modes, and is given componentwise by (4), while the shift operator $\mathcal{S}$ simply multiplies the $k$-th mode by $(-1)^{k}$. We thus again write our fully nonlinear problem as $(6)$, where we now regard $\mathcal{F}$ as being defined on the state

$$
U=(\bar{P}, \bar{V}, \underline{P}, \underline{V})=\left(\left\langle\bar{p}_{j}\right\rangle,\left\langle\bar{v}_{j}\right\rangle,\left\langle\underline{p}_{j}\right\rangle,\left\langle\underline{v}_{j}\right\rangle\right),
$$

each of these being a coefficient vector indexed by $\mathbb{Z}$.

We use the $H^{s}$ norm for solutions to the PDE (2). By the Plancherel identity, this can be taken to be

$$
\|(p, v)\|_{H^{s}}=\|(P, V)\|_{s}=\sqrt{\sum k^{2 s} p_{k}^{2}+k^{2 s} v_{k}^{2}}
$$

where, without loss of generality, but with slight abuse of notation, we have neglected the 0-mode term, which according to (14) is constant. Indeed, because the equations (2), (14) are in conservative form, 0-modes are preserved, which means that our base state is the fixed constant state $\mathbb{1}$ throughout the evolution.

Having described the norms, we now approximate the full nonlinear operator $\mathcal{F}$ with a mollified $\mathcal{F}_{N}$. Rather than the usual Friedrichs mollification, it is easier to use a simple Fourier cutoff: that is, we obtain the approximation $\mathcal{F}_{N}$ by ignoring all modes beyond the $N$-th. This is analogous to (but simpler than) the mollification used by Tadmor in [11]. First, in (2), 
we obtain our smoothed equations by projecting onto modes $k$ with $k \leq N$ : that is, if $\Pi_{N}$ is the projection, so that

$$
\Pi_{N}\left[\sum w_{k} e^{i k t}\right]=\sum_{|k| \leq N} w_{k} e^{i k t},
$$

then our smoothed evolution operator $\mathcal{E}_{N}$ is given by the projected system

$$
p_{x}^{N}+v_{t}^{N}=0, \quad v_{x}^{N}-\Pi_{N}\left[q\left(p^{N}\right)\right]_{t}=0,
$$

where again $q(p)=\gamma p^{-1 / \gamma}$, and differentiation commutes with projection. Expressing this in terms of coefficient vectors, and again using $\Pi_{N}$ to denote the projection, so that

$$
\Pi_{N}[W]=\Pi_{N}\left[\left\langle w_{k}\right\rangle\right]=\left(w_{-N}, \ldots, w_{N}\right),
$$

then the corresponding projected ODE system is

$$
P^{N^{\prime}}=\boldsymbol{D}^{N} V^{N}, \quad V^{N^{\prime}}=\boldsymbol{D}^{N} \Pi_{N} Q\left(P^{N}\right),
$$

where $\boldsymbol{D}^{N}=\operatorname{diag}\{-N, \cdots, N\}$ and $Q$ is given as above.

Having projected the evolution operator $\mathcal{E}$, it is now trivial to obtain the projection of $\mathcal{F}$, namely

$$
\mathcal{F}_{N}=\hat{\mathcal{E}}_{N}-\hat{\mathcal{J}}
$$

since it is clear that both the jump $\mathcal{J}$ and shift $\mathcal{S}$ commute with the projection $\Pi_{N}$.

The Fourier cutoff thus allows us to replace a fully nonlinear PDE with a finite system of ODEs. This demonstrates one of the major strengths of the Nash-Moser method, namely by suitably smoothing the nonlinear operator, we obtain a much simpler object on which to try to derive the appropriate estimates. Indeed, in our case, the hardest estimate, that of inverting nearby linearized operators, is reduced to the problem of inverting an $N \times N$ matrix.

\section{Assumed Bounds}

We now explicitly state the bounds which we will need to show that the Nash-Moser iteration converges. We state these assumptions for an abstract operator $\mathcal{F}$, which has been approximated by $\mathcal{F}_{N}$, and $N$ is assumed to be large. Note that we do not require that the approximation $\mathcal{F}_{N}$ be finite dimensional. We also allow $\mathcal{F}$ and $\mathcal{F}_{N}$ to depend on one or more parameters $\theta$, although for clarity we keep this dependence implicit, and we have fixed an appropriate norm.

We make the following assumptions, which should be valid for all $U$ in some compact neighborhood of our base state, say

$$
\|U-\mathbb{1}\| \leq \delta \leq \frac{1}{2}
$$

and uniformly in parameters unless otherwise stated. In each of the following assumptions, there exist some constant $K_{i}>0$ and exponent $p_{i} \geq 0$ for which the given inequality holds. 
First, we require that the approximation $\mathcal{F}_{N}$ is $C^{1}$ and satisfies a Taylor estimate, namely

$$
\left\|\mathcal{F}_{N}(U-X)-\mathcal{F}_{N}(U)+D \mathcal{F}_{N}(U)[X]\right\| \leq K_{1} N^{p_{1}}\|X\|^{2} .
$$

Here $p_{1}$ counts the number of derivatives lost in the Taylor expansion.

Next, the approximations must converge at some positive rate,

$$
\left\|\mathcal{F}_{N}(U)-\mathcal{F}(U)\right\| \leq K_{2} N^{-p_{2}}
$$

which can be equivalently stated by comparing $\mathcal{F}_{N}(U)$ and $\mathcal{F}_{N+\tilde{N}}(U)$ since we are working in a Banach space.

If we choose to approximate the linearized map $D \mathcal{F}_{N}(U)$ by some $\mathcal{A}_{N}$, this approximation must be controlled,

$$
\left\|\mathcal{A}_{N}(U)-D \mathcal{F}_{N}(U)\right\| \leq K_{3} N^{-p_{3}}
$$

This approximation could arise if one were to neglect lower order terms, say, which is often done in the study of hyperbolic systems [8]. If we choose not to approximate, then $\mathcal{A}_{N}=D \mathcal{F}_{N}$, and we can take $p_{3}=\infty$.

Finally, we need bounds for the nearby linearized operators,

$$
\left\|\mathcal{A}_{N}(U)^{-1}\right\| \leq K_{4} N^{p_{4}}
$$

where we again allow a loss of $p_{4}$ derivatives. In general we do not expect this estimate to hold uniformly because of the presence of resonances, so this is the step in which we expunge parameters. That is, we require (A4) to hold for for $\mathcal{A}_{N}(U)=\mathcal{A}_{N}(U, \theta)$, and for $\theta$ outside of some set $\mathcal{N}$ satisfying

$$
\mu(\mathcal{N}) \leq K_{5} N^{-p_{5}}
$$

where $\mu$ is Lebesgue measure on the parameter space.

\section{Convergence}

We now assume the estimates (A1)-(A5) and prove the convergence of the Nash-Moser iteration, subject to compatibility constraints on the exponents $p_{i}$. The iteration is given by (8), (9) and (10), so we must describe the $m$-th approximations $\mathcal{F}_{m}$ and $\mathcal{A}_{m}$ : we do this by using the Fourier cutoff, by setting

$$
\mathcal{F}_{m}=\mathcal{F}_{N_{m}} \quad \text { and } \quad \mathcal{A}_{m}=\mathcal{A}_{N_{m}}
$$

where $\left\{N_{m}\right\}$ is a sequence, to be described below, which increases to $\infty$.

We must also begin the iteration, by describing the initial guess $U_{0}$. To do this, recall that the constant state $\mathbb{1}$ is a trivial solution of the nonlinear problem, and the linearization of $\mathcal{F}$ around that solution has a one-mode solution $Z$, provided (7) holds. Choosing a small amplitude $\alpha$, we begin the iteration with the initial guess

$$
U_{0}=\mathbb{1}+\alpha Z_{0}
$$

where $Z_{0}$ is the 1-mode solution corresponding to a fixed value of the parameter, say $\theta_{0}$, so that

$$
D \mathcal{F}\left(\mathbb{1} ; \theta_{0}\right)\left[Z_{0}\right]=0,
$$


and with $\left\|Z_{0}\right\|=1$, say.

We are now in a position to state our main theorem.

Theorem 1. Suppose that estimates (A1)-(A5) hold for some constants $K_{i}>0$ and exponents $p_{i}$ which satisfy the compatibility conditions

$$
p_{1}+2 p_{4}<p_{2}, \quad p_{4}<p_{3}, \quad \text { and } p_{4}<p_{5} .
$$

Then there is some $\alpha_{0}>0$, such that for any $\alpha<\alpha_{0}$, there is a set $\mathcal{G}_{\alpha}$ of positive measure, such that for $\theta \in \mathcal{G}_{\alpha}$, the iteration beginning with initial guess (16) converges to a nontrivial solution $U \approx U_{0}$, satisfying

$$
\mathcal{F}(U ; \theta)=0 .
$$

The compatibility conditions (17) can be interpreted as follows: the first expresses the fact that there is enough smoothing that the loss of derivatives in $\mathcal{F}$ and $\mathcal{A}_{m}^{-1}$ can be controlled; the second that our approximation $\mathcal{A}_{m}$ is close enough to the linearization $D \mathcal{F}_{m}$ that the inverse is an effective one; and the third says that we cannot excise too much of the parameter space to get an effective inverse. As noted earlier, if we can invert $D \mathcal{F}_{m}$ without approximation, we can take $p_{3}=\infty$, and similarly if we can invert without excising parameters, we can take $p_{5}=\infty$.

Proof. Once we have chosen the initial iteration $U_{0}$ using (16), we again allow $\theta$ to vary. We have $Y_{0}=\mathcal{F}_{0}\left(U_{0}\right)=\mathcal{F}_{0}\left(U_{0} ; \theta\right)$, and we use (A1) to estimate

$$
\begin{aligned}
\left\|Y_{0}\right\|= & \left\|\mathcal{F}_{0}\left(\mathbb{1}+\alpha Z_{0} ; \theta\right)-\mathcal{F}_{0}(\mathbb{1})-\alpha D \mathcal{F}_{0}\left(\mathbb{1} ; \theta_{0}\right)\left[Z_{0}\right]\right\| \\
\leq & \left\|\mathcal{F}_{0}\left(\mathbb{1}+\alpha Z_{0} ; \theta\right)-\mathcal{F}_{0}(\mathbb{1})-\alpha D \mathcal{F}_{0}(\mathbb{1} ; \theta)\left[Z_{0}\right]\right\| \\
& \quad+\alpha\left\|D \mathcal{F}_{0}(\mathbb{1} ; \theta)\left[Z_{0}\right]-D \mathcal{F}_{0}\left(\mathbb{1} ; \theta_{0}\right)\left[Z_{0}\right]\right\| \\
\leq & K_{1} N_{0}^{p_{1}} \alpha^{2}+\alpha C_{0}\left|\theta-\theta_{0}\right| \\
\leq & 2 K_{1} N_{0}^{p_{1}} \alpha^{2}
\end{aligned}
$$

provided that $\theta$ is restricted to the set

$$
\left|\theta-\theta_{0}\right| \leq \frac{K_{1}}{C_{0}} N_{0}^{p_{1}} \alpha
$$

Here the constant $C_{0}$ is a Lipschitz constant for the function

$$
\theta \mapsto D \mathcal{F}_{0}(\mathbb{1} ; \theta)\left[Z_{0}\right] \in \mathbb{R}^{4},
$$

since $D \mathcal{F}(\mathbb{1})$ respects the 1 -mode vector $Z_{0}$.

Now suppose by way of induction that

$$
\left\|Y_{m}\right\| \leq N_{m}^{-q},
$$

for some $q \geq 0$ to be determined. Then from (11), (12), we have

$$
\begin{aligned}
\left\|Y_{m+1}\right\| & \leq\|I\|+\|I I\|+\|I I I\| \\
& \leq 2 K_{2} N_{m}^{-p_{2}}+K_{3} N_{m}^{-p_{3}}\left\|X_{m+1}\right\|+K_{1} N_{m}^{p_{1}}\left\|X_{m+1}\right\|^{2}
\end{aligned}
$$


where we have used (A2), (A3) and (A1), respectively. Now using (A4) we estimate

$$
\left\|X_{m+1}\right\| \leq K_{4} N_{m}^{p_{4}} N_{m}^{-q},
$$

for $\theta \notin \mathcal{N}_{m}$, and substituting in we get

$$
\begin{aligned}
\left\|Y_{m+1}\right\| & \leq 2 K_{2} N_{m}^{-p_{2}}+K_{3} K_{4} N_{m}^{-\left(q+p_{3}-p_{4}\right)}+K_{1} K_{4}^{2} N_{m}^{-\left(2 q-2 p_{4}-p_{1}\right)} \\
& \leq N_{m}^{-r},
\end{aligned}
$$

provided we choose

$$
r<\min \left\{p_{2}, q+p_{3}-p_{4}, 2 q-2 p_{4}-p_{1}\right\},
$$

and take $N_{0}$ to be large enough. Thus our induction hypothesis is verified if

$$
N_{m}^{-r} \leq N_{m+1}^{-q}, \quad \text { that is } \quad N_{m+1} \leq N_{m}^{r / q} .
$$

It remains to choose $\alpha$, exponents $q$ and $r$, and the sequence $\left\{N_{m}\right\}$ in such a way that convergence is guaranteed. First, assuming $r>q$, and with $N_{0}$ to be chosen, we set

$$
N_{m+1}=\left\lfloor N_{m}^{r / q}\right\rfloor,
$$

where $\lfloor\cdot\rfloor$ is the floor function, so that $(23)$ holds for all $m$. It follows inductively that $N_{m}$ grows rapidly,

$$
N_{m}=O\left(N_{0}^{(r / q)^{m}}\right),
$$

and in particular, we can sum the series

$$
\sum N_{m}^{-\nu} \leq C(\nu) N_{0}^{-\nu}
$$

for some constant $C(\nu)=O(1)$, for any $\nu>0$.

We can choose $r>q$ consistently provided (22) holds, so we ask that each of

$$
q<p_{2}, \quad q<q+p_{3}-p_{4}, \quad \text { and } \quad q<2 q-2 p_{4}-p_{1}
$$

hold; eliminating $q$, we thus require that

$$
p_{4}<p_{3} \text { and } p_{1}+2 p_{4}<p_{2},
$$

which are the first two conditions in (17).

We now consider the choice of $\alpha$ and $N_{0}$. First, according to (18), (20), to start the induction we require

$$
K_{1} N_{0}^{p_{1}} \alpha^{2} \leq N_{0}^{-q}, \quad \text { that is } \quad \alpha \leq \frac{N_{0}^{-\left(q+p_{1}\right) / 2}}{\sqrt{K_{1}}} .
$$

We must also show that our iteration converges to a nontrivial solution. To this end, we use (21) and (25) to estimate

$$
\sum\left\|X_{m+1}\right\| \leq \sum K_{4} N_{m}^{-\left(q-p_{4}\right)}=C\left(q-p_{4}\right) N_{0}^{-\left(q-p_{4}\right)} .
$$

Thus if we choose

$$
C\left(q-p_{4}\right) N_{0}^{-\left(q-p_{4}\right)}<\alpha,
$$

then

$$
\left\|\sum X_{m+1}\right\|<\alpha=\left\|\alpha Z_{0}\right\|
$$


so that

$$
\lim U_{m}=U_{0}-\sum X_{m+1}=\mathbb{1}+\alpha Z_{0}-\sum X_{m+1} \neq \mathbb{1},
$$

and the limiting solution is nontrivial. Combining (26) and (27), we require

$$
C\left(q-p_{4}\right) N_{0}^{-\left(q-p_{4}\right)}<\frac{N_{0}^{-\left(q+p_{1}\right) / 2}}{\sqrt{K_{1}}},
$$

which holds for $N_{0}$ large enough as long as

$$
q-p_{4}>\left(q+p_{1}\right) / 2, \quad \text { which is } q>2 p_{4}+p_{1},
$$

and which is guaranteed by (22).

Finally we recall that at each iteration step, the set $\mathcal{N}_{m}$ of near-resonant $\theta$ values was excluded. Thus the iteration is defined only for $\theta \notin \cup \mathcal{N}_{m}$, which by (A5), (25) has measure

$$
\mu\left(\bigcup \mathcal{N}_{m}\right) \leq \sum \mu\left(\mathcal{N}_{m}\right) \leq \sum K_{5} N_{m}^{-p_{5}}=C\left(p_{5}\right) K_{5} N_{0}^{-p_{5}}
$$

Thus, according to (19), we will retain a set of positive measure in the limit provided

$$
C\left(p_{5}\right) K_{5} N_{0}^{-p_{5}}<\frac{K_{1}}{C_{0}} N_{0}^{p_{1}} \alpha .
$$

Thus in choosing $\alpha$, we require (26), (27) and

$$
\frac{C_{0} C\left(p_{5}\right) K_{5}}{K_{1}} N_{0}^{-\left(p_{5}+p_{1}\right)}<\alpha,
$$

so $\alpha$ can be consistently chosen if $N_{0}$ is large enough and

$$
p_{5}+p_{1}>\left(q+p_{1}\right) / 2, \quad \text { that is } q<p_{1}+2 p_{5},
$$

and which together with $(28)$ requires $p_{4}<p_{5}$, which is the remaining condition in (17).

Here we have chosen $\alpha$ depending on the value of $N_{0}$; evidently for fixed $\alpha$ small enough, we can instead choose $N_{0}$ so that the same conditions are satisfied. By doing so, we get convergence for $\alpha$ small enough. This completes the proof of the theorem.

\section{REFERENCES}

1. A. Bressan, The unique limit of the Glimm scheme, Arch. Rat. Mech. Anal. 130 (1995), 205-230.

2. A. Bressan, T.P. Liu, and T. Yang, $L_{1}$ stability estimates for $n \times n$ conservation laws, Arch. Rat. Mech. Anal. 149 (1999), 1-22.

3. K. Deimling, Nonlinear Functional Analysis, Springer-Verlag, 1985. XIV, 450 S., 35 Abb., DM 98 (ISBN: 978-0387139289).

4. J. Glimm, Solutions in the large for nonlinear hyperbolic systems of equations, Comm. Pure Appl. Math. 18 (1965), 697-715.

5. J. Glimm and P.D. Lax, Decay of solutions of systems of nonlinear hyperbolic conservation laws, Memoirs Amer. Math. Soc. 101 (1970).

6. Fritz John, Formation of singularities in one-dimensional nonlinear wave propagation, Comm. Pure Appl. Math. 27 (1974), 377-405. 
7. Tai-Ping Liu, Decay to $N$-waves of solutions of general systems of nonlinear hyperbolic conservation laws, Comm. Pure Appl. Math. 30 (1977), 585-610.

8. A. Majda, Compressible fluid flow and systems of conservation laws in several space variables, Applied Mathematical Sciences, no. 53, Springer-Verlag, 1984.

9. Jurgen Moser, A new technique for the construction of solutions of nonlinear differential equations, Proc. Nat. Acad. Sci. 47 (1961), 1824-1831.

10. B. Riemann, The propagation of planar air waves of finite amplitude, Classic Papers in Shock Compression Science (J.N. Johnson and R. Cheret, eds.), Springer, 1998.

11. Eitan Tadmor, Convergence of spectral mathods for nonlinear conservation laws, SIAM J. Num. Anal. 26 (1989), 30-44.

12. B. Temple and R. Young, The large time stability of sound waves, Comm. Math. Phys. 179 (1996), 417-466.

13. Blake Temple and Robin Young, A paradigm for time-periodic sound wave propagation in the compressible Euler equations, Methods and Appls of Analysis 16 (2009), no. 3, $341-364$.

14. _ A Liapunov-Schmidt reduction for time-periodic solutions of the compressible Euler equations, Methods and Appls of Analysis 17 (2010), no. 3, 225-262.

15. _ Time-periodic linearized solutions of the compressible Euler equations and a problem of small divisors, SIAM Journal of Math Anal 43 (2011), no. 1, 1-49.

16. Robin Young, Sup-norm stability for Glimm's scheme, Comm. Pure Appl. Math. 46 (1993), 903-948.

Department of Mathematics, University of California, Davis, CA 95616

Department of Mathematics and Statistics, University of Massachusetts, Amherst, MA 01003 